\title{
Was symbolisieren die bestimmten Artikel des Deutschen?*
}

\section{Fragestellung}

Eine wesentliche morpho-syntaktische Eigenschaft pronominaler Formen ist ihre Kongruenz mit dem Nomen. In den Grammatiken werden die pronominalen Paradigmen deshalb anhand der Kategorien des Nomens konstruiert. So wird traditionellerweise im Deutschen für all die verschiedenen pronominalen Elemente wie bestimmter/unbestimmter Artikel, Negationsartikel, Possessiv- und Demonstrativpronomen, starke/ schwache Adjektive ein und dieselbe Struktur des Paradigmensystems zugrundegelegt. Die 3 Genusklassen konstituieren je ein Paradigma im Singular sowie ein gemeinsames Pluralparadigma. Jedes dieser 4 Paradigmen hat 4 Kasuspositionen, Nom., Gen., Dat., Akk. Dies ergibt ein Paradigmensystem mit 16 Paradigmenpositionen. Jede Position beschreibt eine der möglichen syntaktischen Umgebungen von nominalen Einheiten auf der Äußerungsoberfläche. Nicht nur im Deutschen existiert nun aber keineswegs für jede dieser Positionen auch eine eigenständige pronominale Form. Die Diskrepanz ist bekanntlich beachtlich. Das Paradigmensystem des bestimmten Artikels - das hier exemplarisch diskutiert werden soll - weist mit 6 Formen noch den größten Formenreichtum auf. Das Demonstrativpronomen dies und der Negationsartikel kein z.B. haben 5 distinkte Formen, die schwachen Adjektive schließlich nur 2.

Die Frage, die sich unmittelbar aufdrängt, ist, welche (grammatische) Ratio steckt hinter diesem hohen Maß an Formidentitäten. Inwieweit haben wir es hier mit motivierten Synkretismen, d.h. auf inhaltlich begründeten Neutralisationen beruhenden Formidentitäten, und/oder zufälligen Homonymien zu tun? Im Hinblick auf die pronominalen Paradigmen des Deutschen sind dieser Frage meines Wissens bisher Bierwisch (1967), Blevins (1995) und Wiese (1997) nachgegangen. Auch in Plank (1991) wird die Behandlung von Synkretismen in der Morphologie von verschiedenen Autoren für verschiedene Sprachen angesprochen. Ich will jedoch nur auf die zuerst genannten Arbeiten kurz eingehen. Mit explizitem Verweis auf Jakobson und Hjelmslev unternimmt Bierwisch (1967) im Rahmen eines generativen Grammatikmodells die Übertragung der Theorie der distinktiven Merkmale und ihrer Neutralisation sowie der Markiertheitstheorie aus der Phonologie auf die Beschreibung von flexionsmorphologischen Distinktionen.

\footnotetext{
Der Beitrag beruht auf den nach zweijähriger Publikationsdauer in Bittner (2001) erschienenen ersten Überlegungen zu diescm Thema und deren Weiterentwicklung, die mit verschiedenen Schwerpunkten auf dem Workshop, Genus als grammatische Kategorie' am 11./12.6.1999 in Bamberg, dem 9. International Morphology Meeting vom 25.- 27.2.00 in Wien sowie der ,Conference on Gender and Inflectional Class' am 27.5.00 in Tromsö vorgestellt wurden. Ich danke allen Diskussionspartnern für ihre Anregungen, besonders Andreas Bittner, Wolfgang Ullrich Dressler, Livio Gaeta, Bernhard Hurch, KlausMichael Köpcke, Elisabeth Leiss, Trond Trosterud, Barbara Unterbeck, Doris Weber und Bernd Wiese.
} 
Blevins (1995) argumentiert anhand der Erfassung motivierter Synkretismen für die Annahme einer hierarchischen und paradigmatischen Organisation des Lexikons sowie unterspezifizierter Paradigmen. Die sich aus der (unumgänglichen) Mitlieferung von Interpretationsinformation auf der syntaktischen Ebene ergebende Unterspezifizierbarkeit von Information auf der morphologischen Ebene ist schließlich ein Grundgedanke bei Wiese (1997), der außerdem auf die Beteiligung der phonologischen Struktur der Affixe an der (ikonischen) Kodierung der Merkmalsinhalte hinweist. Trotz der unterschiedlichen Zielstellungen und Argumentationswege ist allen drei Arbeiten der Versuch gemeinsam, die Formidentitäten auf Neutralisationen syntaktischer Merkmale zurückzuführen. Es wurde also - etwas verkürzt dargestellt - danach gefragt, ob sich Synkretismen dadurch begründen, daß etwa Nom. und Akk. als unmarkierte Kasus auf der morphologischen Ebene undifferenziert bleiben und sich von den übrigen Kasus durch Negativspezifikation wie [-oblique/-governed] oder Nichtrelevanz bestimmter Kasusmerkmale wie [+oblique] unterscheiden. Während Bierwisch dabei mit positiv und negativ spezifizierten Merkmalen arbeitet, sind bei Blevins und Wiese nur positiv spezifizierte Merkmale relevant. Immer werden jedoch spezifische Kasusmerkmale (Bierwisch: \pm oblique, Igoverned; Blevins: oblique, nonoblique, dative, accusative; Wiese: oblique, object) und spezifische Genusmerkmale (Bierwisch: \pm Masc., \pm Femin.; Blevins: nonfemin., masc.; Wiese: standard, special) unterschieden, bei Bierwisch auch noch spezifische Numerusmerkmale ( \pm Plur.). Die Zielstellung, die Paradigmen anhand einer optimalen Anzahl von Merkmalen, d.h. möglichst ökonomisch und elegant $\mathrm{zu}$ beschreiben, führte zur Ansetzung von je zwei Merkmalen für die Genus- und Kasusdifferenzierung sowie einem Merkmal zur Differenzierung der beiden Numeri bei Bierwisch. Da es sich bei diesen Merkmalen jedoch um syntaktische Merkmale handelt, Merkmale also, die die Äußerungsstruktur betreffen, und nicht um Merkmale, die den Informationsgehalt, den semiotischen Inhalt der grammatischen Formen beschreiben, ist m.E. die Frage, welche Ratio hinter den Formidentitäten in synkretistischen Paradigmen liegt, noch nicht beantwortet.

Gemeinsame syntaktische Merkmale bzw. gleiches syntaktisches Verhalten von sprachlichen Einheiten stellen zwar wichtige Indizien für die Erklärung von Synkretismen dar, sind aber genau wie diese selbst eher als Folge einer Informationsidentität zu betrachten, denn als ihre Ursache. Die hier angesprochene Problematik der gewählten distinktiven Merkmale wird im Genusbereich besonders deutlich: Die zur Differenzierung der 3 Genera herangezogenen Kriterien sind im Prinzip willkürlich bestimmbar. Ob man nun [+masc] und [+fem] oder [+fem] und [+ntr] oder wie Wiese [standard] und [spezial] als differenzierende Merkmale wählt, bleibt letztlich gleich, solange nicht klar ist, welche auf die Interpretation der Aussage bezogenen Informationen sich hinter diesen Merkmalen verstecken. Auch Annahmen über Markiertheitsverhältnisse, mit denen die Wahl der Merkmale motiviert wird, müssen unabhängig begründet und bewiesen, m.a.W. auf außersprachliche Merkmale zurückgeführt werden. Das gleiche gilt im Grunde für die Systematisierung der Kasusdifferenzierungen. Was sagen uns Merkmale wie [+object] oder [+oblique] über den Beitrag, den eine Kasusform zur Interpretation der Äußerung liefert; insbesondere dann, wenn es mehrere Formen für einen Kasus gibt?

Interessant ist, daß der Unterschied zwischen strukturellen Merkmalen (Verhalten in Lautverbindungen) und distinktiven Merkmalen (Artikulationsart und -ort) in der Phonologie von Beginn an berücksichtigt wurde. Im Unterschied zum Phonemsystem, 
das auf artikulatorischen Gegebenheiten beruht, beruht die Morphologie auf semiotischen Gegebenheiten, d.h. auf der Kodierung von Information mittels Zeichen. Distinktive Merkmale auf morphologischer Ebene müssen somit den semiotischen Gehalt des sprachlichen Zeichens wiedergeben. Sie können folglich nur semantische Merkmale sein. Um welche Art von semantischen Merkmalen es sich dabei handeln dürfte, ist vor allem von Peirce und in dessen Rezeption von Jakobson (1957) deutlich gemacht worden. Jakobson (1965) hat zudem auf den langen historischen Vorlauf der Peirce'schen Überlegungen zu , deixis' und ,interpreter' verwiesen. In die Linguistik nach Jakobson sind die Aspekte dieser zeichentheoretischen Überlegungen zwar eingegangen und zur Beschreibung grammatischer Strukturen insbesondere zur Beschreibung von Kategorien und Klassen (etwa als \pm deiktisch, \pm telisch, tholistisch, \pm definit) relevant geblieben, sehr aus dem Blickfeld geraten ist aber für längere Zeit, daß mit diesen Merkmalen bzw. den Kategorien, die sie tragen, grammatische Techniken verbunden sind. D.h., daß jede Grammatik mit ihren Kategorien ein System bereitstellt, diese Merkmale auf jeder Ebene, für jeden Aspekt einer sprachlichen Äußerung variieren zu können. Wie verschieden die grammatischen Systeme zum Ausdruck gleicher Merkmalskonstellationen konstruiert sein können, haben inzwischen u.a. die Arbeiten des Kölner Universalienprojektes (s. u.a. Seiler/Lehmann 1982; Seiler/Stachowiak 1982; Seiler 1986) oder auch Gil (1991) oder Leiss (2000) deutlich gemacht. Es kann auf diesem Hintergrund durchaus als begründet angesehen werden, ein begrenztes Sample an semantischen Merkmalen anzunehmen, die in jeweils sprachspezifischer Kombination und Verortung der Grammatik aller Sprachen zugrundeliegen.'

Die Frage, wie sich die Formidentitäten in den pronominalen Paradigmen erklären, sei hiermit also nochmals gestellt. Ziel der vorliegenden Untersuchung ist es, eine Annäherung an die Merkmale zu erreichen, die die Symbolisierungsleistung der verschiedenen Formen des bestimmten Artikels charakterisieren. Als methodische Maximalannahme lege ich meiner Analyse das Prinzip ,one function - one form ' zugrunde; im hochfunktionalen Bereich der grammatischen Formen sollten Homonymien unerwünscht sein. Die entsprechende Maximalthese der Untersuchung ist: Jede der 6 Formen des bestimmten Artikels besitzt genau eine Funktion. Sie besteht in der Symbolisierung eines bestimmten Komplexes von Interpretationshinweisen. Dieser kann durch semantische Merkmale beschrieben werden.

\section{Formale Analyse des Paradigmensystems}

Ganz im Sinne des Prinzips ,one function-one form ' und der Annahme, daß Formales (Hinweise auf) Funktionales birgt, soll eine formale Analyse des Paradigmensystems Ausgangspunkt der Betrachtungen sein. Eine nur geringe Abweichung von der traditionellen Anordnung der Paradigmen ergibt eine Darstellung, in der ein überraschendes $\mathrm{Maß}$ an Symmetrie in der Verteilung der 6 Artikelformen gegeben ist:

1 Für eine inspirierende Einführung in diese hier nur kurz angedeutete Auffassung von Grammatik vgl. Leiss (1992: 1-11). 
Abb. 1: ,traditionelles“ Paradigmensystem

\begin{tabular}{|l|c|c|c|c|}
\hline \multirow{2}{*}{} & \multicolumn{3}{|c|}{ Singular } & Plural \\
\cline { 2 - 4 } & Femin. & Mask. & Neutr. & \\
\hline Nom. & die & der & das & die \\
\hline Gen. & der & des & des & der \\
\hline Dat. & der & dem & dem & den \\
\hline Akk. & die & den & das & die \\
\hline
\end{tabular}

Die 4 die-Formen liegen an den 4 Eckpunkten, die 4 der-Formen sowie die 2 das- und die 2 den-Formen liegen an den äußeren Kanten der Darstellung und die 2 des- und die 2 dem-Formen bilden das Zentrum. Macht man diese symmetrische Verteilung durch Einrahmungen graphisch deutlich, ergeben sich 3 voneinander abgegrenzte Bereiche: der Bereich der die-Formen, der Bereich der der-, das- und den-Formen und der Bereich der des- und dem-Formen.

Abb. 2: „symmetrisches" Paradigmensystem

\begin{tabular}{|c|c|c|c|c|}
\hline & Femin. & Mask. & Neutr. & Plural \\
\hline Nom. & die & der & das & die \\
\hline Gen. & der & des & des & der \\
\hline Dat. & der & dem & dem & den \\
\hline Akk. & die & den & das & die \\
\hline
\end{tabular}

Die gefundene Konstellation läßt sich mengentheoretisch interpretieren: Wir haben vor uns die Menge aller bestimmten Artikel. Innerhalb dieser Menge gibt es zwei Teilmengen, wobei die kleinste Teilmenge wiederum Teilmenge der ersten Teilmenge ist. In Merkmalen ausgedrickt heißt das: Es gibt mindestens ein Merkmal, das alle bestimmten Artikel besitzen. Es gibt desweiteren mindestens ein Merkmal, das die der-, desund den-Formen von den die-Formen unterscheidet und es gibt mindestens ein weiteres Merkmal, das nur die des- und dem-Formen besitzen. Merkmale, die alle bestimmten Artikel besitzen können wir als Basismerkmale (BM) und Merkmale, die die Teilmengen konstituieren, als spezifische Merkmale (Spec1 + Spec2) bezeichnen. Rein formal betrachtet, weisen also die die-Formen nur Basismerkmale auf, die der-, das- und den-Formen Basismerkmale und spezifizierende Merkmale der ersten Stufe und die desund dem-Formen enthalten die Basismerkmale, die spezifizierenden Merkmale der ersten Stufe und die spezifizierenden Merkmale der zweiten Stufe, vgl.:

$$
\begin{array}{ll}
\text { die } & \text { BM } \\
\text { der, den, das } & B M+\text { Spec1 } \\
\text { des, dem } & B M+\text { Spec1 + Spec2 }
\end{array}
$$

Die gleiche Darstellung, die wir gerade mengentheoretisch interpretiert haben, läßt sich (in vorsichtiger Anlehnung an Jakobsons Merkmalswürfel zum russischen Kasussystem, Jakobson 1958) auch geometrisch interpretieren. Klappt man die äußeren Ränder/Felder der Darstellung in Abb. 2 nach hinten um (man muß dabei die Eckfelder nach innen einklappen), erhält man letztlich einen Würfel (dazu muß man sich die Eckfelder wieder 
ausgeklappt vorstellen, so daß sie die Bodenfläche bilden). Dieser Würfel hat drei Ebenen, eine Grundebene (Bodenfläche) eine mittlere Ebene (Seitenwände) und eine obere Ebene (Deckfläche). Auf der Grundfläche befinden sich die 4 die-Formen, auf der mittleren Ebene die der-, das- und den-Formen und auf der oberen Ebene die des- und dem-Formen. Interpretiert man dies (wiederum in vorsichtiger Anlehnung an Jakobson) als Struktur mit von unten nach oben zunehmender inhaltlicher Komplexität, verhält sich das Ergebnis analog zur mengentheoretischen Hypothese: Alle 4 die-Formen befinden sich auf der Basisebene, auf der nächsten, inhaltlich komplexeren Ebene liegen die der-, das- und den-Formen und auf der folgenden, inhaltlich komplexesten Ebene befinden sich die des- und dem-Formen.

Soweit vorerst zu den funny games, die sich aus der überraschend symmetrischen Verteilung der Artikelformen ableiten lassen. Es stellt sich die Frage: Bedeutet das alles etwas? Kommt man auf diesem Weg zu Erkenntnissen über den funktionalen Charakter der verschiedenen Artikelformen und über funktionale Hintergründe der Formidentitäten? Möglicherweise ist die gefundene Anordnung ein völlig bedeutungsloses Artefakt der gewählten Paradigmenschreibweise. Aber auch das wäre zu beweisen. Wir wollen zunächst annehmen, daß sie unbedingt bedeutungsvoll ist.

\section{Kategoriale Analyse des Paradigmensystems}

Nähern wir uns dem Form-Funktions-Problem weiter von der syntaktischen Oberfläche her und betrachten zunächst die die-Formen. Die erscheint sowohl im Singular - und zwar der Femin. - als auch im Plural, und es erscheint sowohl im Nom. als auch im Akk. Die Symbolisierungsleistung von die kann daher weder + Femin. noch + Sg., noch + Pl. sowie weder + Nom. noch + Akk. sein. Es symbolisiert keine dieser Kategorien eindeutig. Die Aussage läßt sich noch verschärfen: Wenn die im Singular und im Plural steht, ist es für keine der beiden Numeruskategorien signifikant, aus demselben Grund ist es auch nicht für Femin. signifikant. D.h., die Form die spezifiziert weder einen Numerus noch ein Genus. Hinsichtlich Kasus könnte man Bierwisch (1967) folgend annehmen, daß das Merkmal [+direkt] symbolisiert wird, welches Nom. und Akk. den beiden obliquen Kasus Gen. und Dat. gegenüberstellt. Allerdings sind Nom. und Akk. außer im Mask, vollkommen identisch (s. Abb. I und 2). Und das nicht nur bei den bestimmten Artikeln, sondern mit Ausnahme der Personalpronomen in allen pronominalen Paradigmen. Nom. und Akk. bilden im Deutschen die morphologisch unmarkierten Kasus. Da die unmarkierten Teile von Oppositionen typischerweise keine Merkmale spezifizieren (Jakobson 1936), gehen wir davon aus, daß [+direkt] nicht als relevantes Merkmal zu betrachten ist. D.h., die übermittelt nicht nur keine Genus- und Numerusinformation, die symbolisiert auch keine spezifische Kasusinformation.

Doch worin liegt die Symbolisierungsleistung von die? - Das Merkmal, das alle bestimmten Artikel teilen, ist, bestimmter Artikel zu sein. Mit dem bestimmten Artikel wird als Information transportiert: Der Sprecher setzt voraus, daß dem Hörer das aktuelle Referenzobjekt bekannt ist. Wir können dies mit dem Merkmal [+bekannt] 
umschreiben. Im Sinne unserer mengentheoretischen Interpretation des Paradigmensystems kann [+bekannt] als Basismerkmal aller bestimmten Artikel betrachtet werden. Es ist zugleich das einzige im Moment erfaßbare Merkmal, das durch die Artikelform die symbolisiert wird. Auch Bierwisch nimmt mit [+det] (+determiniert) ein allen Demonstrativpronomen gemeinsames Merkmal an. Bei Blevins und Wiese fehlt dagegen ein solches Merkmal. Die jeweils unmarkierteste Form bleibt in beiden Untersuchungen merkmallos (bei Blevins: die und keine; bei Wiese die und seine). Welche Funktion, welchen Symbolisierungsgehalt diese Formen haben, die ja grammatische Formen sind und daher keine lexikalische Semantik aufweisen, bleibt hier offen.

Die Untersuchung, welche Kategorien durch die symbolisiert werden, läßt sich umkehren in eine Untersuchung, welche der schon betrachteten Kategorien überhaupt eindeutig durch eine bestimmte Artikelform symbolisiert wird. Es zeigt sich, daß es keine Artikelform gibt, die eindeutig Nom. oder Akk. symbolisiert, sowie keine Artikelform, durch die eindeutig Plural symbolisiert wird. Alle Formen des Pluralparadigmas erscheinen auch in den Singularparadigmen. Wir nehmen dies zum Anlaß, die Kategorienangaben Singular, Plural, Femin., Nom. und Akk. aus unserer Darstellung des Paradigmensystems zu streichen. Was bleibt? Erhalten bleiben die Angaben Mask., Neutr., Gen. und Dat. - jedoch keine Numerusangabe! Die Kategorie Numerus ist durch die bestimmten Artikel offenbar nicht repräsentiert. Sind die bestimmten Artikel numerusindifferent? Auch Blevins und Wiese haben darauf verwiesen, daß eine Numerusangabe im unterspezifizierten Paradigma nicht notwendig ist, mit der Begründung, daß das Auftreten eines Genusmerkmals Singular impliziert, während das Nichtauftreten eines Genusmerkmals Plural impliziert. Dazu muß man aber wissen bzw. anderweitig im Merkmalssystem verankern, daß diese Implikationen gelten. D.h., der Existenz einer Numerusdistinktion muß so letztlich doch Rechnung getragen werden. Viel interessanter ist in diesem Zusammenhang das Greenbergsche Universal (Greenberg 1980), das besagt, daß das Vorhandensein von Genus immer das Vorhandensein von Numerus voraussetzt. Genus scheint demnach eine Extension von Numerus zu sein. Die Behandlung dieser Frage soll jedoch auf Abschn. 3 verschoben werden. Machen wir uns zuvor die verbleibende Kategorienkonstellation des Paradigmensystems graphisch deutlich:

Abb. 3: kategorial "reduzicrtes" Paradigmensystem

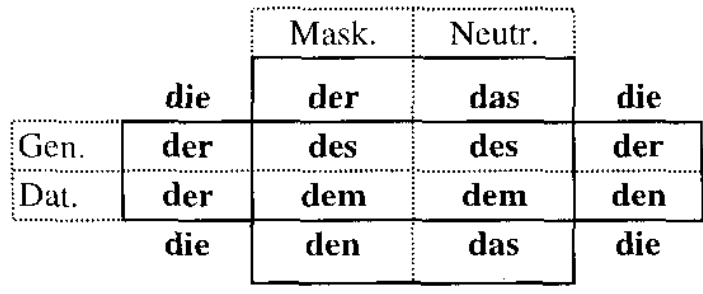

Interpretiert man das Verbleiben von Mask. und Neutr. sowie Gen. und Dat. merkmalstheoretisch, so ergibt sich, daß mit diesen Kategorien eine spezifische(re) Information verbunden sein muß, als mit den nicht mehr aufscheinenden Kategorien. Daß gerade Gen. und Dat. als spezifischere Kasus gegenüber Nom. und Akk. erscheinen, verwundert nicht. Sie gelten traditionell als die markierteren oder inhaltlich komplexeren Kasus. Überraschend ist, daß im Genusbereich Mask. und Neutr. als die spezifischeren Genera erscheinen. Im allgemeinen wird doch das Mask. als das unmarkierte Genus des 
Deutschen betrachtet und wenn nicht das Mask., dann wäre (so z.B. Bierwisch 1967) das Neutr. als unmarkiert anzusehen - aber nicht das Femin. Hier sieht es jedoch so aus, als würden sich Femin. und Plural im Genusbereich wie Nom. und Akk. im Kasusbereich verhalten. Diese Parallelität wird aber nicht nur durch die erfolgte Kategorientilgung suggeriert, sondern auch formal durch die jeweiligen Artikelformen! Nom. und Akk. unterscheiden sich lediglich im Mask., wo der vs. den auftritt, und Femin. und Plural unterscheiden sich lediglich im Dat., wo wiederum der vs. den auftritt. Die Genusklassifikation erweist sich einmal mehr als ein Rätsel.

Abb. 3 enthält einen weiteren interessanten Aspekt. Es wird nicht nur augenscheinlich, daß das Paradigmensystem letztlich eine Kreuzklassifikation von Genus und Kasus darstellt, vielmehr ergibt sich, daß sich beide Klassifikationen gerade im Bereich der traditionell als markiert(er) angesehenen des- und dem-Formen kreuzen. Die Markiertheit dieser Formen könnte also aus der Summierung von Genus- und Kasusmerkmalen resultieren. Die Analogie zu unserer mengentheoretischen Interpretation der Formenverteilung im Paradigmensystem (s. Abb. 2 und darunter) ist auffällig.

In der weiteren Analyse der Markierungs- und Markiertheitsverhältnisse im Paradigmensystem wenden wir uns zunächst den Fragen zu, die mit der Genusklassifikation verbunden sind, und begeben uns dazu zunächst wieder ganz in die Hand der grammatischen Formen.

\section{Die Genusfragen}

Aus den in Abschn. 2 festgestellten formalen Parallelitäten ergeben sich bei Ansetzung der Maximalthese folgende Fragen:

1. Warum ist das Paradigma der Femin. fast formgleich mit dem Pluralparadigma? Welche gemeinsamen Eigenschaften haben Femin. und Plural?

2. Warum besitzen Mask. und Neutr. identische Formen in Gen. und Dat.? Welche gemeinsamen Eigenschaften haben Mask. und Neutr.?

Die Fragen zeigen, daß wir uns mit den Hintergründen der Genusklassifikation der Nomina beschäftigen müssen. Untersuchungen zur Motiviertheit des Genus bei den deutschen Nomina (vgl. u.a. Köpcke 1982, Köpcke/Zubin 1996, Corbett 1991) haben vornehmlich den Bereich der Simplizia als Herausforderung betrachtet. Der Bereich der Derivativa gilt als nicht vergleichsweise interessant. Hier liegt eine weitgehend eindeutige Korrelation des Genus mit dem Ableitungssuffix vor. Alles erscheint klar geregelt. Für die Frage, welcher Inhalt/welche Information durch die Genusklassifikation kodiert wird bzw. mit ihr assoziiert ist, zeigen sich aber gerade hier aufschlußreiche Konstellationen: Alle Suffixe, die Femin. ableiten, bilden (mit Ausnahme des Movierungssuffixes -in) Abstrakta. Alle Suffixe, die Mask. ableiten, bilden im wesentlichen Nomina agentis und Nomina instrumenti, d.h. Konkreta. Es gibt nur wenige Suffixe, die ausschließlich Neutr. bilden; lediglich das Diminutivsuffix -chen und die Kollektivkonstruktionen mit Präfix ge- und Wortausgang/e/. Der weitaus überwiegende Teil der derivierten Neutr. 
wird durch Konversion erzeugt (Vogel 1996). Diese Bildungen sind reine Substantivierungen, sie behalten den kontinuativen Charakter der Ableitungsbasen bei.

Abb. 4 Korrelation von Genus und Wortbildung

\begin{tabular}{|c|c|c|c|}
\hline & FEMININ & MASKULIN & NEUTRUM \\
\hline $\begin{array}{l}\text { unabgeleitete } \\
\text { Nomen }\end{array}$ & $\begin{array}{l}\text { Ente } \\
\text { Wand } \\
\text { Dauer }\end{array}$ & $\begin{array}{l}\text { Alfe } \\
\text { Mut } \\
\text { Garten }\end{array}$ & $\begin{array}{l}\text { Auge } \\
\text { Haus } \\
\text { Kissen } \\
\end{array}$ \\
\hline $\begin{array}{l}\text { abgeleitete } \\
\text { Nomen }\end{array}$ & $\begin{array}{l}\frac{\text {-heit/-keit: }}{\text { Freiheit }} \\
\text { Regsamkeit } \\
\text {-schaft: } \\
\text { Leidenschaft } \\
\text { Landschaft } \\
\text {-ung: } \\
\text { Kleidung } \\
\text { Überlegung } \\
\text {-ei: } \\
\text { Quälerei } \\
\text { Brauerei } \\
\text {-ik: } \\
\text { Theatralik } \\
\text { Mimik } \\
\text {-ion: } \\
\text { Diskussion } \\
\text { Perfektion } \\
\text {-ie: } \\
\text { Therapie } \\
\text { Prosodie } \\
\text {..................................... }\end{array}$ & $\begin{array}{l}\text {-er: } \\
\text { Turner } \\
\text { Bohrer } \\
\text { ling: } \\
\text { Lehrling } \\
\text { Säugling } \\
\text { Setzling } \\
\text {-ist: } \\
\text { Telefonist } \\
\text { Traktorist } \\
\text {-or: } \\
\text { Doktor } \\
\text { Autor } \\
\text {-ent/ant: } \\
\text { Konsument } \\
\text { Sekundant } \\
\text {-erich/-ian/-ikus: (unprod.) } \\
\text { Wüterich } \\
\text { Grobian } \\
\text { Pfiffikus } \\
\text {... }\end{array}$ & $\begin{array}{l}\text { KONVERSION: } \\
\text { das Blau } \\
\text { das Tief } \\
\text { das Armsein } \\
\text { das In-Gebrauch-Kommen } \\
\text { das Laufen } \\
\text { das Nachdenken } \\
\text { das Wenn-und-Aber } \\
\text { das Aua } \\
\text {-nis: } \\
\text { Gefängnis } \\
\text { Ärgernis } \\
\text { Bedürnis } \\
\text { ge- (e): } \\
\text { Gebirge } \\
\text { Gerenne } \\
\text { Geschenk } \\
\text { Gebrüll } \\
\text {-tum: (unproduktiv) } \\
\text { Wachstum } \\
\text { Eigentum } \\
\text {..................................................................... }\end{array}$ \\
\hline $\begin{array}{l}\text { prototypische } \\
\text { Klasse }\end{array}$ & Abstrakta & $\begin{array}{l}\text { Individuativa } \\
\text { Konkreta }\end{array}$ & Kontinuativa \\
\hline
\end{tabular}

Es besteht also eine klare Zuordnung von Femin. - Abstraktum, Mask. - Konkretum und Neutr. - Kollektivum/Kontinuativum. Im Bereich der Derivation gibt es offenbar eine semantische Basis für die Genuszuordnung. Außerdem scheint das Genus hier in gewissem Maße als paradigmatische Kategorie wirksam zu sein. Man kann von ein und derselben Basis Ableitungen in alle drei Genusklassen herstellen, vgl.:

$\begin{array}{llll}\text { krank } & \text { fischen } & \underline{\text { rot }} & \underline{\text { schnell }} \\ \text { die Krankheit } & \text { die Fischerei } & \text { die Röte/Rotheit } & \text { die Schnelle/Schnelligkeit } \\ \text { der Krankelein Kranker } & \text { der Fischer } & \text { der Rotelein Roter } & \text { der Schnelle/ein Schneller } \\ \text { das Kranke } & \text { das Fischen } & \text { das Rot } & \text { das Schnelle }\end{array}$

Diese paradigmatische Eigenschaft hat das Genus im Bereich der nichtderivierten Nomen offenbar verloren, es ist dort eine erstarrte Kategorie.

Leiss (1997) macht darauf aufmerksam, daß im Ahd. möglicherweise noch die ursprüngliche paradigmatische Kraft des Genus aufspürbar ist. Für einen Großteil der Nomen, die in den ahd. Wörterbüchern mit wechselndem Genus belegt sind, liegen zugleich verschiedene Bedeutungsangaben vor. Leiss' erste Systematisierung dieser Angaben ergab, daß offenbar femin. Genus mit kollektiver Bedeutung, mask. Genus mit 
singulativer Bedeutung und neutr. Genus mit kontinuativer Bedeutung korreliert. Mit einigen kleinen Verschiebungen entspricht dieser ahd. Befund den heutigen Verhältnissen in der Wortbildung der Nomina. Es sieht so aus, als hätte die Wortbildung die Funktion des Genus übernommen ohne ganz die Bindung zum Genus zu verlieren bzw. als wäre Genus grammatikalisierte Wortbildung.

$\mathrm{Daß}$ es sich bei den konstatierten Korrelationen der Genera mit den angeführten Merkmalen nicht um eine sprachinterne Spekulation handelt, wird deutlich, wenn man einen Blick in die Genusliteratur des 19. Jahrhunderts wirft. Vergleichbare Korellationen sind bereits häufig und vor allem auch cross-linguistisch festgestellt worden, vgl. Weber (1999).

Die Frage nach der Gemeinsamkeit von Femin. und Plural kann auf diesem Hintergrund umformuliert werden in die Frage:

1a. Was verbindet Abstrakta und Kollektiva mit Plural?

Dies ist bereits an verschiedenen Stellen dargelegt worden und auch Leiss' Artikel zum Genus im Ahd. enthält einen entsprechenden Hinweis: Kollektiva werden als nondistributive Plurale charakterisiert. Kollektivbildungen fassen mehrere Entitäten derselben Art zu einem Ganzen zusammen. Abstrakta sind ähnlich, sie fassen Entitäten unterschiedlicher Art zu einem Ganzen zusammen. Beiden Wortklassen ist gemeinsam, daß sie eine Zusammenfassung von Einzelelementen beinhalten und darin wiederum liegt ihre Gemeinsamkeit mit dem Plural. Nun sind im Nhd. nur die Abstrakta Femin. und als solche weitgehend formgleich mit dem Plural. Die Neutralisation der distinktiven Merkmale muß also so angelegt sein, daß sich kein Zusammenfall mit den Kollektiva ergibt. Kollektiva unterscheiden sich von Abstrakta und Plural darin, daß bei ihnen das Ganze, die Objekthaftigkeit des Bezeichneten hervorgehoben wird. Sie bilden ein begrenztes Ganzes. Abstrakta und Plural dagegen bezeichnen Dinge, deren äußere Grenzen nicht relevant sind. Abstrakta und Plural treffen sich in ihrer Eigenschaft unbegrenzte Ganze zu sein.

Die ahd. Mask. werden als Singulativa charakterisiert. Im Nhd. sind mask. Derivativa Bezeichnungen für Individuativa und Konkreta. Sowohl Singulative als auch Individuativa und Konkreta repräsentieren begrenzte unteilbare Ganze.

Bleiben die Neutr.! Fürs Ahd. sind sie als Mass Nouns und Kontinuativa charakterisiert worden. Im Nhd. sind neutr. Derivativa, wie schon festgestellt, vorrangig Kontinuativa und Kollektiva. Die gemeinsame Eigenschaft dieser beiden Wortklassen ist, daß sie Dinge bezeichnen, die sich aus Entitäten derselben Art konstituieren. Dies könnte die Basis sein, auf der sie sich in der Entwicklung vom Ahd. zum Nhd. einander angenähert haben. Gegenwärtig ist zu beobachten, daß auch Ableitungen mit Präfix geund Wortausgang auf /e/ Kontinuativcharakter annehmen, vgl. Gerede, Gelache, Gefahre. Kontinuativa sind unabgeschlossene Ganze, so wie Abstrakta und Plural. Ihre Spezifik ist dabei, daß bei ihnen nicht nur das Ganze, sondern auch die Teile unabgeschlossen sind. Jede Ausgrenzung aus einer kontinuativen Struktur ist wieder eine kontinuative Struktur, mit exakt denselben Eigenschaften wie die Ausgangsgröße. Das gilt nicht für Abstrakta, die quasi emergente Ableitungen sind, und nicht für Plural. Eine ihrer Einzelentitäten ergibt nicht wieder einen Plural oder ein Abstraktum. Zusammenfassend können wir also die These aufstellen, daß mit den einzelnen Genusklassen heute noch resthaft folgende inhaltlichen Aspekte verknüpft sind: 


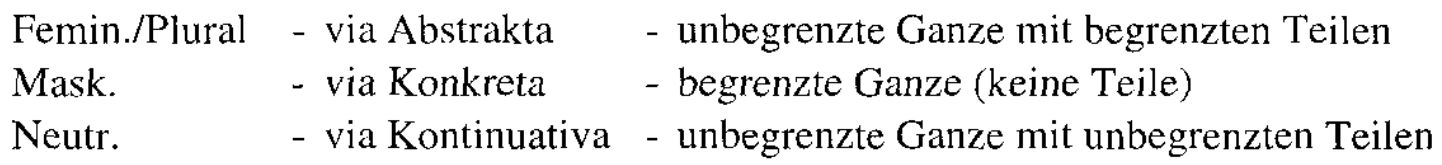

Für die weitere Analyse der Artikelformen ist nun ein wichtiger Unterschied zwischen dem Ahd. und dem Nhd. zu beachten. Im Ahd. bzw. in noch früheren Sprachperioden resultierten die distinktiven Merkmale der Genusklassen, ihr kategoriales Potential (vermutlich) aus den Teil-Ganzes-Klassifikationen. Im Nhd. dagegen ist Genus - wie bereits festgestellt - eine grammatikalisierte Reliktkategorie. Für jedes Nomen ist ein bestimmtes Genus festgeschrieben. Die originäre Funktion des Genus, die Symbolisierung der spezifischen Teil-Ganzes-Struktur des Referenzobjekts, wurde von der Wortbildung übernommen, die nur noch sekundär mit den Genusklassen verbunden ist. Die Genusmarkierung selbst wurde vom Wortende des Nomens auf die pronominalen Elemente, primär die Artikel, verlagert. Sie ist jetzt kombiniert mit den originären Eigenschaften dieser Elemente. Eine wesentliche Eigenschaft der Artikel, sowohl des unbestimmten als auch des bestimmten Artikels ist, daß sie die Referenz auf einen begrenzten, als Ganzes wahrnehmbaren Gegenstand symbolisieren. In den heutigen Genusklassen ist also die Symbolisierung einer bestimmten Teil-Ganzes-Struktur der Referenzsemantik der jeweiligen pronominalen Formen, im Fall der Artikel also der Referenz auf begrenzte Ganze, untergeordnet. Die Überlagerung von Informationen verschiedener Bereiche in den pronominalen Einheiten - hinzu kommen mindestens noch Kasusinformationen - hat einerseits zur Folge, daß die jeweiligen Informationsanteile für den Linguisten schwer zu erschließen sind, andererseits ist sie wohl zugleich die Basis für umfangreiche Neutralisationen, die den drohenden Informationsüberfluß auf das notwendige Maß begrenzen sollen.

Aus den obigen Überlegungen ergibt sich folgende These: Die weitgehende Formgleichheit von Femin. und Plural steht im Zusammenhang mit gemeinsamen Eigenschaften in der Teil-Ganzes-Struktur der in beide Klassen eingeordneten Nomen. Es handelt sich jeweils um unbegrenzte Ganze, die sich aus begrenzten Teilen konstituieren. Im Hinblick auf die nur bei Femin. und Plural auftretende Artikelform die ist jedoch festzuhalten, daß es nicht zu den unmittelbaren Eigenschaften von die gehört diese TeilGanzes-Struktur zu symbolisieren. Dies ergibt sich aus dem Auftreten anderer Artikelformen in den Paradigmen der Femin. und des Plurals. Die Symbolisierung der TeilGanzes-Struktur , unbegrenztes Ganzes mit begrenzten Teilen' kann maximal als sekundäre Eigenschaft der Artikelform die betrachtet werden. Es sei noch einmal betont: Die jeweiligen Teil-Ganzes-Verhältnisse bzw. Perspektivierungen werden nicht durch das grammatische Potential der bestimmten Artikel erzeugt, sondern entweder durch Wortbildungselemente oder durch die Pluralmarkierung. Der bestimmte Artikel die symbolisiert primär lediglich das Merkmal [+bekannt].

Wir kommen nun zur Diskussion der zweiten Genusfrage: Warum besitzen Mask. und Neutr. identische Formen in Gen. und Dat.? Welche gemeinsamen Eigenschaften haben Mask. und Neutr.?

Wie die obige Diskussion der über die Wortbildung an die Genusklassen assoziierten Teil-Ganzes-Eigenschaften gezeigt hat, lassen sich für Mask. und Neutr. keine vergleichbaren Gemeinsamkeiten feststellen. Mask. korreliert mit der Struktur, begrenztes (unteilbares) Ganzes', Neutr. dagegen mit der Struktur , unbegrenztes Ganzes mit unbe- 
grenzten Teilen'. Ein Blick auf das gemeinsame Paradigmensystem von unbestimmtem Artikel, Possessiv- und Negationsartikel, in dem auch der Nom. beider Genera identisch ist, d.h., die einzige Distinktion im Akk. besteht, verstärkt jedoch die Erwartung gemeinsamer Eigenschaften, Aber machen wir uns zunächst einen weiteren Unterschied bewußt. Wirkliche Kontinuativa können nicht bzw. nur unter spezifischen Kontextbedingungen mit dem unbestimmten Artikel verbunden werden: *ein Wasser, *ein Sand, *ein Rot, *ein Laufen. Wären alle Neutr. Kontinuativa würde diese Genusklasse im Bereich des unbestimmten Artikels nicht existieren. Sie erscheint nur/erst im Bereich [+bestimmt] bzw. genauer [+bekannt]. Das Merkmal [+bekannt] enthält inhärent das Merkmal [+begrenzt]. Bekannt im Sinne textstrukturierender Bekanntheit sind stets konkrete begrenzte Entitäten bzw. Gesamtheiten/Totalitäten. Wenn also ein kontinuativer Ausdruck mit einem bestimmten Artikel oder auch einem Possessiv- oder Negationsartikel verbunden wird, wird die Referenz auf einen begrenzten Teil oder den gesamten Umfang der jeweiligen Entität symbolisiert. M.a.W. die Existenz der Klasse der Neutr. beruht - via Kontinuativa - auf der Begrenzung eines ,unbegrenzten Ganzen'. Mask. sind - via Konkreta - von sich aus , begrenzte Ganze'. Über die Konstatierung des unterschiedlichen Verhaltens beim unbestimmten Artikel - konkret der Nichtexistenz

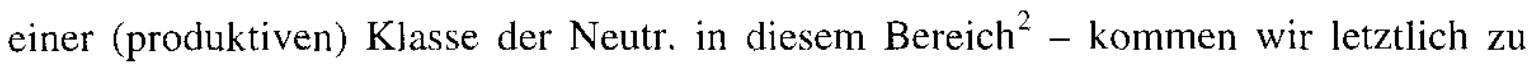
einer These über Gemeinsamkeiten von Mask. und Neutr.: Mask. und Neutr. repräsentieren ,begrenzte Ganze', d.h. sie haben das gemeinsame Merkmal [+begrenzt]. Gleichzeitig liefern die unterschiedlichen Teil-Ganzes-Strukturen auch die Basis für eine These über ein distinktives Merkmal beider Genera: Während Mask. nichtteilbare Ganze sind, sind Neutr. teilbare Ganze. Das Merkmal [+teilbar] begründet die formale Distinktion in Nom. und Akk., scheint aber in Dat. und Gen. neutralisiert zu sein.

Wir müssen an dieser Stelle auf Femin. und Plural zurückkommen. Auch für diese Klassen gilt, daß die Verbindung mit dem bestimmten Artikel die Referenz auf eine bekannte und damit begrenzte Entität symbolisiert. Doch die durch den Artikel die hergestellte Begrenztheit stellt bei Abstraktum und Plural die Präsenz der Teile, also die auf Vielheit beruhende Konstellation nicht in den Hintergrund. Mask. und Neutr bzw. die der-/das-Paradigmen könnten somit die eindeutig singulären Klassen sein, während die beiden die-Paradigmen, also Femin. und Plural, numerusunspezifisch sind. In Jakobsons Terminologie hieße das, daß sich die Form die als einzige Form, die nicht im Paradigma der Mask. und der Neutr. erscheint, zum Merkmal [+begrenzt] (im Unterschied zu [+bekannt]) unspezifisch oder neutral verhält. Sie sagt über die An- oder Abwesenheit dieses Merkmals nichts aus; was unsere obige Aussage wiederholt.

Die in Abschn. 2 aufgeworfene Frage nach dem Zusammenhang von Genus und Numerus ist indirekt schon beantwortet. Beide Kategorien dienen der Perspektivierung der Gestalteigenschaften des nominalen Referenten. Die Differenzierung des Singulars in die verschiedenen Genusklassen stellt zusätzliche Perspektivierungsmöglichkeiten zur Verfügung. Aus dem festgestellten Zusammenhang von Genus und Wortbildung

2 Es ist hier wieder wichtig zwischen der herausgearbeiteten Korrelation von Neutr. mit Kollektiva und der grammatikalisierten Klasse der Neutr. zu unterscheiden. Wenn man annehmen kann, daß dort, wo diese Grammatikalisierung nicht vorliegt, das neutr. Genus produktiv dic mit dem Terminus, Kollektivum' bezeichnete Teil-Ganzes-Struktur crzeugt, gibt es keine Klasse der Neutr. im Bercich des unbestimmten Artikels. 
läßt sich vermuten, daß Sprachen ohne Genus diese zusätzlichen Perspektivierungsmöglichkeiten durch die Wortbildung erhalten.

\section{Genusbasiertes Zwischenergebnis}

Welche Merkmalszuschreibungen sind nach der Erörterung der Genusfragen nun möglich und wie sieht das Paradigmensystem aus? Es ist relativ klar oder schon klarer, welche Symbolisierungsleistung die Formen die und das besitzen. Außerdem wissen wir noch etwas über die Form der im Bereich der Genusklassifikation.

Abb. 5: „Merkmalssynthese“ von bestimmtem Artikel und Genus

\begin{tabular}{|c|c|c|c|c|}
\hline 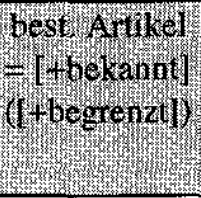 & 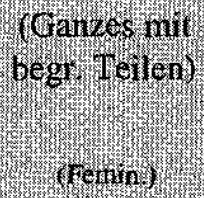 & begn Ganres & 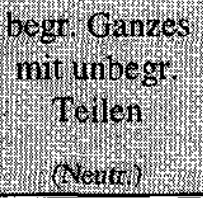 & 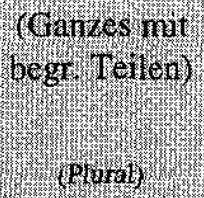 \\
\hline & die & $\operatorname{der}$ & Thas & orre \\
\hline Genitiv & der & des & des & der \\
\hline \multirow[t]{2}{*}{ Dativ } & der & dem & dem & den \\
\hline & ore & $\begin{array}{c}\text { den } \\
\|\|\|\|\|\|\end{array}$ & das & \\
\hline
\end{tabular}

Folgende vorläufige Merkmalszuschreibungen zu den Artikeln können vorgenommen werden:

$\begin{array}{llll}\text { die } & {[+ \text { bekannt }]} & & \\ \text { der/Mask. } & {[+ \text { bekannt }]} & {[+ \text { begrenzt }]} & \\ \text { das } & {[+ \text { bekannt }]} & {[+ \text { begrenzt }]} & \text { [+teilbar }]\end{array}$

Offen bleiben drei der-Formen, die den-Formen und die des- und dem-Formen. Die Darstellung macht deutlich, daß wir uns der Kasusklassifikation zuwenden müssen. Alle noch nicht ergründeten Formen liegen, bis auf eine der beiden den-Formen, im Bereich der spezifischen oder markierten Kasus. Wiederum sollen der Maximalthese ,one function - one form' folgend die aus den Formidentitäten resultierenden Kasusfragen gestellt werden. 


\section{Die Kasusfragen}

Analog zu den Genusfragen stellen sich im Kasusbereich die folgenden Fragen:

1. Was haben Nom. und Akk. gemeinsam? Warum gibt es im Mask. einen (den einzigen) Unterschied zwischen Nom. und Akk.?

2. Was unterscheidet Nom. und Akk. von Dat. und Gen.?

3. Was haben Dat. und Gen. gemeinsam und worin unterscheiden sie sich?

Die erste Frage ist relativ schnell beantwortet. Nom. und Akk. sind die morphologisch unspezifizierten Kasus. Während der Nom. generell als der unmarkierte Kasus zu betrachten ist, d.h., er kodiert keine spezifische Information (vgl. auch Jakobson 1936 zum Russ.), steht der Akk. dem Nom. in der Funktion gegenüber, das vom Verbereignis direkt betroffene Argument zu enthalten. Dieser Unterschied wird aber im deutschen Basissatz zum einen durch die Wortstellung und zum anderen durch die konzeptuellen Wahrscheinlichkeiten realisiert. Erst wenn diese Präsuppositionen verletzt wird, müssen weitere Symbolisierungsmittel wie z.B. der Kontrastakzent eingesetzt werden. Aus den genannten Gründen kann der Akk. im Deutschen wie in vielen anderen Sprachen morphologisch unmarkiert bleiben. Für das Auftreten von den im Akk. der Mask. gibt es ebenfalls eine allgemein akzeptierte Erklärung. Sie baut genau auf dem eben Gesagten auf. Mask. sind die typischen Agenskandidaten. Warum das so ist, ist durch die aufgezeigte Korrelation von Mask. mit Ableitungen für [+human]-Bezeichnungen (also bevorzugte Agenskandidaten) unterstrichen worden. Erscheint ein typischer Agenskandidat, d.h. ein Mask., als Patiens bzw. vom Verbereignis Betroffener, so wird gegen eine Präsupposition verstoßen. Genau in diesen Fällen tritt Grammatik ein, d.h., eine bestimmte grammatische Form/Struktur, in diesem Fall den, zeigt das Abweichen von der Erwartung an.

Die zweite Frage kann man zunächst ganz allgemein beantworten. Gen. und Dat. markieren spezifischere Verhältnisse des jeweiligen Arguments zum Verbereignis. ${ }^{3}$ Läßt man sich zur Beantwortung der Frage, was die in diesen Kasus auftretenden bestimmten Artikel symbolisieren, von den Formen leiten, so ist zunächst festzustellen, daß alle Formen, die im Gen. und im Dat. erscheinen auch im Mask. und im Neutr. erscheinen. Wenn die Formidentitäten Inhaltsidentitäten repräsentieren sollte dies etwas bedeuten. Eine besondere Schwierigkeit stellt die Form den dar. Auch in den von Bierwisch, Blevins und Wiese vorgestellten Analysen konnten die beiden den-Formen nicht vereinigt werden. Wie schon festgestellt, verhindern genau die den-Formen die Zusammenfassung der Paradigmen von Nom./Akk. sowie Femin./Plural. Während das Auftreten von den im Akk. der Mask. plausibel erklärbar ist, erschließt sich das Auftreten von den im Dat.Plural nicht ohne weiteres. Hinsichtlich der Architektur des Paradigmensystems haben wir die gleiche Situation zwischen Gen.Fem./PI. und Dat.Fem./Pl. wie zwischen Mask.Nom./Akk. und Neutr.Nom./Akk. (vgl. Abb. 3), wobei im ersten Fall alle vier Formen zusammenfallen könnten, träte nicht die den-Form auf. Die entsprechende Frage soll also analog formuliert werden: Warum wird der Plural im Dat. spezifisch markiert?

3 Für eine detaillierte Untersuchung und inhaltliche Begründung der „Rangordnung“ zwischen Gen.-, Dat.- und Akk.-Objekten vgl. Primus (1999). 
Eine Spielerei mit der Symmetrie des Paradigmensystems gibt hier eine Idee und erhellt gleichzeitig die Frage nach den - für die bestimmten Artikel relevanten! - Inhalten der Kasusklassifikation. Dreht man das waagerechte "Kreuzteil" des Paradigmensystems so, daß es auf das senkrechte zu liegen kommt, fallen die Artikelformen des Dat. auf die des Mask. und die des Gen. auf die des Neutr.

Abb. 6: formale „Unifizierung“ von Dat. und Mask. sowie von Gen. und Neutr.

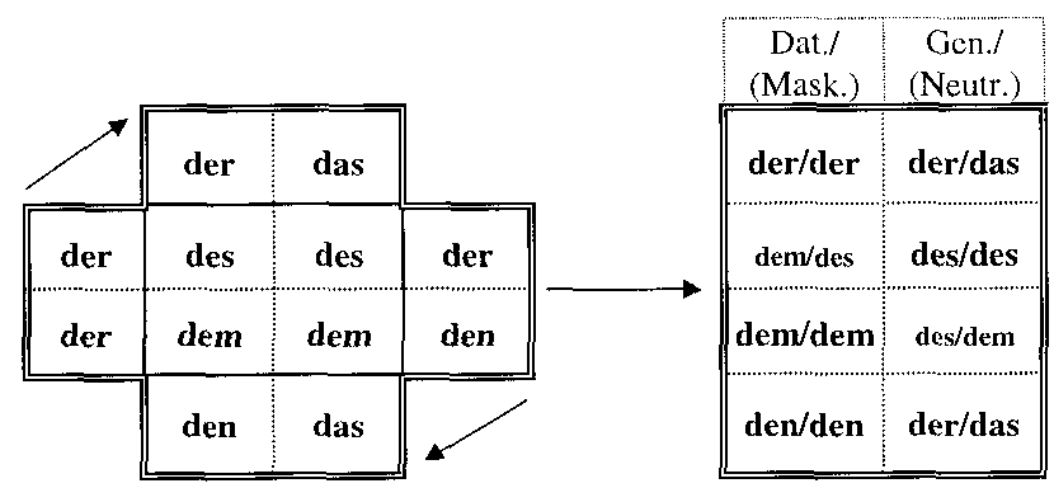

Bei Mask./Dat, fallen dabei nicht nur die den-Formen, sondern auch die der-Formen aufeinander. Haben die Kategorien Dat./Mask. gemeinsame Eigenschaften? Ein erstes Indiz dafür ist die Tatsache, daß Dativobjekte typischerweise Bezeichnungen für Lebewesen, Individuen also, enthalten. Entitäten, die das Merkmal [+animate] besitzen, besitzen prototypischerweise auch die mekmale [+individuativ]/[+singulativ]. Im Deutschen wie auch in anderen Sprachen ist der Dat. der Kasus, der für den ausgedrückten Sachverhalt mehr oder weniger marginale zusätzliche Informationen bereitstellt. Dativische Satzglieder konkretisieren den Sachverhalt. Der Dat. wird auch als Ruhekasus beschrieben, dessen 'Semantik direkt mit der Resultativität und Stativität der PerfektVerben interagiert' (Drossard 1986). Der Hinweis auf perfektive Eigenschaften macht eine Gemeinsamkeit von Dat./Mask. noch greifbarer. Beide Kategorien korrelieren mit Begrenztheit. Aus diesem Zusammenhang erschließt sich eine Motivation für das Auftreten von den im Plural. Trägt der Dat. prototypisch das Merkmal [+begrenzt] muß die Abweichung von dieser Präsupposition im Plural markiert werden. Die Form den in Dativkontexten könnte demnach das Nichtgegebensein von [+begrenzt] symbolisieren.

Bei Neutr./Gen. fallen mit der Drehung die das-Formen des Neutr. und die derFormen des Gen. aufeinander. Hier scheint sich aus dem Spiel mit den Achsen des Paradigmas kein Hinweis auf Gemeinsamkeit der beiden Kategorien zu ergeben. Folgende Überlegungen sind aber möglich: Das Neutr. hatten wir als Klasse der Kontinuativa charakterisiert, bei denen durch die Verbindung mit dem Artikel die Ausgrenzung eines bestimmten Teils vorgenommen wird. Erst durch diese Ausgrenzung wird die Klasse der Neutr. konstituiert und neben die Mask. gestellt. Die Ausgrenzung eines bestimmten Teils aus einer (homogenen) Masse kann als partitive Struktur betrachtet werden. Partitivität wiederum ist die Eigenschaft, die cross-linguistisch typischerweise durch den Gen. symbolisiert wird. Auch im Deutschen, wo der Gen. nur noch als adverbialer Gen. produktiv ist, wird in den entsprechenden Konstruktionen (die Farbe des Buches, die Gedanken des Kindes) stets ein bestimmter Teilaspekt des Referenten der NP in den Focus genommen. Der Gen. grenzt den Teil eines Gegenstandes aus (oder 
ein), der für den Sachverhalt relevant ist (Jakobson 1936). Die Gemeinsamkeit der beiden Kategorien kommt in der formalen Ähnlichkeit der eindeutig neutr. Form das und der eindeutig genitiv. Form des zum Ausdruck. Im Paradigmensystem der Demonstrativpronomen ist der Unterschied der beiden Formen vollständig aufgehoben. Ein weiteres Indiz für die hier angenommenen Gemeinsamkeiten beider Kategorien.

Wir können also als vorläufiges Fazit festhalten: Der Unterschied zwischen Nom./Akk. und Dat./Gen. besteht darin, daß die beiden letzteren Kasus (typischerweise) anzeigen, daß eine begrenzte Entität am Verbereignis beteiligt ist bzw. im Focus der Referenz der NP steht, während die beiden ersten Kasus über dieses Merkmal nichts aussagen. Auch aus diesem Grund können diese Kasus merkmallos bleiben. Tritt der bestimmte Artikel im Nom. und Akk. auf, so symbolisiert er mit Ausnahme des den im Akk. der Mask. die aktuellen Gestalteigenschaften des Referenten, nicht aber in welcher Form der Referent in Relation zum Verbereignis steht. Diese Funktion der beiden Kasus wird im Deutschen nicht morphologisch sondern syntaktisch (oder auch prosodisch) realisiert. Die Gemeinsamkeit zwischen Gen. und Dat. wurde bereits benannt. Der interne Unterschied zwischen Gen. und Dat. schließlich liegt darin, daß im Gen. das Merkmal der Begrenztheit durch das Merkmal der Partitivität spezifiziert wird.

Versuchen wir an dieser Stelle wieder eine Übertragung der festgestellten Merkmalskonstellationen auf die Darstellung des Paradigmensystems.

\section{Genus-Kasusbasiertes Zwischenergebnis}

Die Hinweise aus den Überlegungen zu den Kasusfragen können analog zu Abb. 5 in die Darstellung des Paradigmensystems aufgenommen werden:

Abb. 7: „Merkmalssynthese" von bestimmtem Artikel, Genus und Kasus

\begin{tabular}{|c|c|c|c|c|}
\hline $\begin{array}{c}\text { best. Artikel = } \\
1+\text { bekannt }] \\
([+ \text { begrenzt }])\end{array}$ & $\begin{array}{l}\text { (Ganzes mit } \\
\text { begr. Teilen) }\end{array}$ & begr. Ganzes & $\begin{array}{c}\text { begr. Ganzes } \\
\text { mit unbegr. } \\
\text { Teilen } \\
\text { (Neutr.) }\end{array}$ & $\begin{array}{l}\text { (Ganzes mit } \\
\text { begr. Teilen) }\end{array}$ \\
\hline \multirow{4}{*}{$\begin{array}{l}{[+ \text { begrenzt }]} \\
{[+ \text { partitiv }]} \\
\text { [+begrenzt }] \\
\text { (individuativ) }\end{array}$} & die & der & CHes & die \\
\hline & der & des & des & der \\
\hline & der & dem & $\operatorname{dem}$ & den \\
\hline & die & ten & Uas & die \\
\hline
\end{tabular}

Die Schattierungen sollen an die eingangs präsentierten Überlegungen zu einer 3Ebenen- und zu einer merkmalstheoretischen Interpretation der formalen Struktur des Paradigmensystems erinnern. Eine inhärente, aber nicht berührte Frage war, wie sich die 
verschiedenen Formen auf Ebene 2 (der, das, den) und auf Ebene 3 (des, dem), für die neben dem Basismerkmal ein bzw. zwei spezifische(re) Merkmale angenommen wurden, durch konkrete Merkmale differenzieren lassen. Wir haben gesehen, daß die den-Form spezifische (unerwartete) Aspekte innerhalb des der-Bereichs symbolisiert, der das allen Formen der Ebene 2 gemeinsame Merkmal [+begrenzt] besitzt. Das gleiche gilt für die das-Form, sie symbolisiert spezifischere (partitive) Aspekte innerhalb des der-Bereichs. Eine ähnliche interne Gliederung weist der dem/des-Bereich auf. Die Form des symbolisiert spezifischere (partitive) Aspekte neben dem gemeinsamen Merkmal [+begrenzt].

Es zeigt sich eine erstaunliche Parallelität in den Merkmalsverhältnissen der Ebenen 2 und 3. Die eingangs auf der rein formalen Interpretation der hier gewählten Darstellung des Paradigmensystems beruhende Feststellung, daß der dem/des-Bereich der Bereich des Paradigmensystems ist, in dem sich Genus- und Kasusklassifikation unmittelbar kreuzen, bekommt damit eine inhaltliche Bedeutung. Die traditionell als komplexere Formen bzw. Formen mit komplexerem Kodierungsgehalt betrachteten Formen dem und des scheinen tatsächlich zu beinhalten, daß die jeweiligen Merkmale in diesen Fällen sowohl im Genus- als auch im Kasusbereich relevant sind, d.h. symbolisiert sein sollen. Die Form dem enthält demnach die Symbolisierung von [+begrenzt] hinsichtlich Genus und [+begrenzt] hinsichtlich Kasus, und die Form das die Symbolisierung von [+begrenzt]/[+partititv] hinsichtlich Genus und [+begrenzt]/[+partitiv] hinsichtlich Kasus.

Auch wenn der gegenwärtige Stand der Untersuchungen mit Sicherheit noch nicht alle Fragen beantwortet und ganz sicher auch noch nicht die endgültige Merkmalsstruktur der bestimmen Artikel gefunden ist, kann die Methode, sich in der Analyse der Inhalte von den Formen leiten zu lassen, wohl als fruchtbar eingeschätzt werden. Im folgenden seien die gegenwärtigen Ergebnisse zusammengefaßt.

\section{Zusammenfassung und Ausblick}

Die Diskussion der aufgrund formaler Identitäten gestellten Genus- und Kasusfragen läßt die Annahme folgender Symbolisierungsleistungen durch die Artikelformen zu:

\begin{tabular}{|c|c|c|c|}
\hline die & [+bekannt] & & \\
\hline der & [+bekannt] & [+begrenzt] & \\
\hline den & [+bekannt] & [+begrenzt] & [+nichtpräsup.] \\
\hline das & [+bekannt] & [+begrenzt] & [+partitiv] \\
\hline dem & [+bekannt] & [+begrenzt] & [+begrenzt] \\
\hline des & [+bekannt] & [+begrenzt] & {$[+$ partititv $]$} \\
\hline
\end{tabular}

Da es sich hier um eine vorläufige Auflistung handelt, deren Basierung erst noch deutlicher abgeklärt werden muß, sollen keine voreiligen Schlußfolgerungen über Markiertheitsverhältnisse in Genus und Kasus gezogen werden. Es sei nur angemerkt, daß hier - entgegen der traditionellen Annahme Mask. sei das unmarkierte Genus - 
gerade Femin. als das unmarkierte Genus erscheint. Gegen dieses Ergebnis wurde häufig das Argument angeführt, daß im Mask. das formal differenzierteste Paradigma vorliegt, was für seine Unmarkiertheit spräche; typischerweise sind die unmarkierten Bereiche stärker differenziert als die markierten. Dem sei entgegnet, daß es daneben auch den Bereich der Belebtheitsbezeichnungen gibt, in dem cross-linguistisch eine Tendenz zu stärkerer Differenzierung festzustellen ist. Das Mask. könnte also auch aus diesem Grund das stärker differenzierte Paradigma besitzen. Genau hierin liegt ja letztlich die Ursache für die stärkere Differenzierung im Akk. und möglicherweise für die Entstehung einer neuen (mask.) Genusklasse, vgl. Eisenberg (2000). Interessant ist, daß gerade die Artikelformen, die quasi typisch mask., d.h. [+begrenzt], sind (der, dem, den), genau die Artikelformen sind, die auch im Dat. vorkommen, der ebenfalls typischerweise Belebtheitsbezeichnungen enthält. Niemand wird aber schlußfolgern wollen, daß der Dat. der unmarkierte Kasus ist. Schließlich sei noch auf Afrikaans verwiesen, das als einzigen bestimmten Artikel den Artikel die (und eben nicht der) behalten hat. Solange über die grammatische Funktion von Genus kein gesichertes Wissen besteht, fehlt letztlich eine geeignete Basis für Markiertheitsannahmen bei den Genera.

Aufgrund der Vorläufigkeit der Untersuchungsergebnisse soll hier keine Darstellung eines unterspezifizierten Paradigmas versucht werden. Ganz explizit soll aber noch einmal betont werden, daß der bisherigen Analyse zufolge im Genusbereich die gleichen Merkmale relevant zu sein scheinen wie im Kasusbereich. Folgende Darstellung, die für a l l e proniminalen Paradigmen gilt, kann dies noch einmal verdeutlichen:

Abb. 8: Gemeinsamkeiten von Kasus und Genus in der Pronominalmorphologic

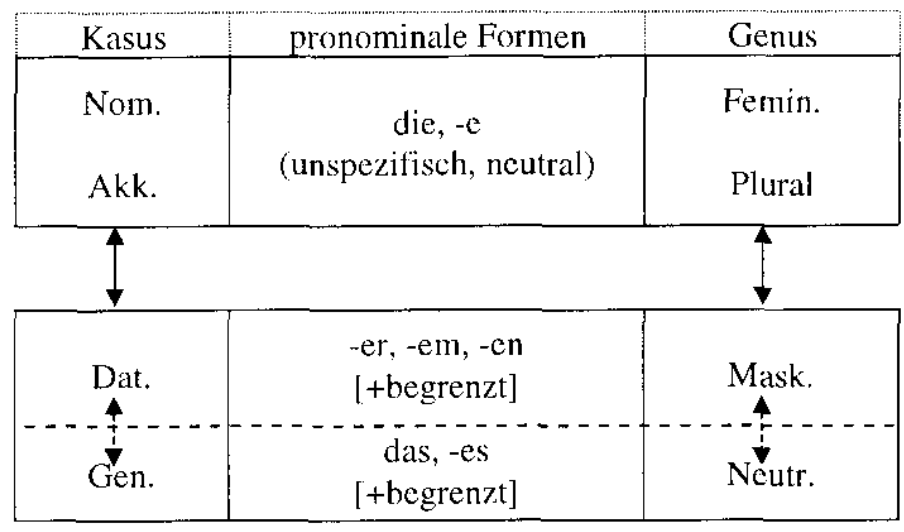

Aus diesen Gemeinsamkeiten auf den beiden kategorialen Ebenen läßt sich die These ableiten, daß die Genusdifferenzierung (einschließlich Numerus) auf der Wortebene dasselbe leistet, was die Kasusdifferenzierung auf der Satzebene leistet. Dies erinnert an Jakobsons These, daß sich die Invarianten der sprachlichen Strukturbildung auf den verschiedenen Ebenen des Sprachsystems wiederholen. In van Schooneveld (1977: 6) findet sich folgende Darstellung dieser Idee gekoppelt mit dem Verweis auf die dahinterliegenden Peirce'schen Merkmalskonzepte, die hier nur eingangs erwähnt, aber noch nicht explizit behandelt worden sind:

Thus, by consistently applying Jakobson's methodology of discounting syntagmatic conditioning and extracting semantic invariants within various categorical molds in a given language, such as parts of speech and lexicon vs. grammar, we can proceed to identify paradigmatic intercategorial 
intralinguistic invariants. These invariants are grouped on various levels of deixis, such as perceptional and transmissional deixis. The result is a paradigmatic structure of great simplicity, yet made complex by its repetitiveness.

Die Analyse hat m.E. gezeigt, daß das Axiom ,one function - one form' ein unbedingt ernst zu nehmendes Prinzip der grammatischen Strukturbildung darstellt. Weitere Untersuchungen sollten zu einem unterspezifizierten Paradigma führen können, das keine Synkretismen enthält und die Distinktionen der Artikelformen mittels semantischer Merkmale erfaßt. Sowohl die Kasus- als auch die Genusbezeichnungen, die das Satzglied auf der syntaktischen Oberfläche beschreiben, sind in einem auf die Informationsleistung der Formen orientierten morphologischen Paradigma überflüssig. In der kombinierten Erfassung der syntaktischen Merkmale/Kontexte und der inneren Systematik der morphologischen Struktur dagegen kann die traditionelle Paradigmenschreibweise, die alle Hinweise auf die tieferen Geheimnisse enthält, als geradezu genial gelten.

\section{Literatur}

Bierwisch, Manfred (1967): Syntactic Features in Morphology: General Problems of So-Called Pronominal Inllection in German. In: To Honour Roman Jakobson. Essays on the Occasion of his Seventieth Birthday /I October 1966. Vol. I. The Haguc/Paris: Mouton. $239-270$

Bittner, Dagmar (2001): The Definite Articles in German - What are the Features Creating an Underspecified Paradigm? In: Rauch, Irmengard; Carr, Gerald F. (eds): New Insights in Germanic Linguistics $/$. Berkeley Insights in Liguistics and Semiotics 38. New York: Lang. I-19

Blevins, James, P. (1995): Syncretism and Paradigmatic Opposition. Linguistics and Philosophy. Volume 18 (6 issues). $113-152$

Corbett, Greville (1991): Gender. Cambridge: Cambridge University Press

Drossard, Werner (1986): Kasusmarkierung und die Zentralität von Partizipanten. Arbeiten des Kölner Universalien-Projekts (akup) 63.1 -28

Eisenberg, Peter (2000): Das vierte Genus? Über die natürliche Kategorisation der deutschen Substantive. In: Bittner, Andreas; Bittner, Dagmar; Köpcke, Klaus-Michael (Hrgg.): Angemessene Strukturen. Systemorganisation in Phonologie, Morphologie und Syntax. Hildesheim: Olms. 91-105

Gil, David (1991): Universal Quantifiers: A Typological Study. Konstanz: Eurotyp/Working paper 12

Greenberg, Joseph H. (1980): Language Universals: with Special Reference to Feature Hierarchies. The Hague: Mouton

Jakobson, Roman (1936/1971): Beitrag zur allgemeinen Kasuslehre. Gesamtbedeutungen der russischen Kasus. In: Selected Writings II. The Hague: Mouton. 23-71

Jakobson, Roman (1957/1971): Shifters, Verbal Categories and the Russian Verb. In: Selected Writings II. The Hague: Mouton. 130-147

Jakobson, Roman (1958/1984): Morfologičeskije nabljudenija nad slavjanskim skloneniem. In: Waugh, Linda; Halle, Morris (eds): Russian and Slavic Grammar: Studies 1931-198/. Berlin: Mouton. 105-134

Jakobson, Roman (1965/1971): Quest for the Essence of Language. In: Selected Writings II. The Hague: Mouton. 345-359

Köpcke, Klaus-Michael (1982): Untersuchungen zum Genussystem der deutschen Gegenwartssprache. Tübingen: Narr (=Linguitische Arbeiten 122)

Köpcke Klaus-Michael; Zubin, David (1996): Prinzipien für dic Genuszuweisung im Deutschen. In: Lang, Ewald; Zifonun, Gisela (Hrgg.): Deutsch typologisch. Jahrbuch des Instituts für deutsche Sprache 1995. Berlin: de Gruyler. 473-491

Leiss, Elisabeth (1992): Die Verbalkategorien des Deutschen. Ein Beitrag zur Theorie der sprachlichen Kategorisierung. Berlin; New York: de Gruyler 
Leiss, Elisabeth (1997): Genus im Althochdeutschen. In: Glaser, Elvira; Schlacler Michael (Hrgg.): Grammatica lanua Artium. Festschrift für Rolf Bergmann zum 60. Geburtstag. Heidelberg: Winter. 33-47

Leiss, Elisabeth (2000): Artikel und Aspekt. Die grammatischen Muster von Definitheit. Berlin; New York: de Gruyter

Peirce, Charles, S. (2000): Semiotische Schriften. Hrsg. u. übers. von Christian Kloesel und Helmut Pape. Frankfurt/Main: Suhrkamp

Plank, Frans (ed.) (1991): Paradigms. The Economy of Inflection. Berlin; New York: Mouton de Gruyter.

Primus, Beatrice (1999): Cases and Thematic Roles. Ergative, Accasative and Active. Tübingen: Niemeyer (=Linguistische Arbeiten 393)

Seiler, Hansjakob (1986): Apprehension: Language, Object and Order. Teil III: The Universal Dimension of Apprehension. Tübingen: Narr (=Language Unviversal series ; 1/III)

Seiler, Hansjakob; Lehmann, Christian (1982): Apprehension: Das sprachliche Erfassen von Gegenständen. Teil I: Bereich und Ordnung der Phänomene. Tübingen: Narr (=Language Unviversal Series; 1/I)

Seiler, Hansjakob; Stachowiak, Franz Josef (1982): Apprehension: Das sprachliche Erfassen von Gegenständen. Teil II: Die Techniken und ihr Zusammenhang in Einzelsprachen. Tübingen: Narr (=Language Unviversal serics ; 1/II)

Van Schooneveld, C. H. (1977): By Way of Introduction: Roman Jakobson's Tenels and their Potential. In: Armstrong, Daniel; van Schooneveld, C. H. (eds.): Roman Jakobson. Echoes of his Scholarship. Lisse: Peter de Ridder Press. 1-11

Vogel, Petra M. (1996): Wortarten und Wortwechsel. Zu Konversion und verwandten Erscheinungen im Deutschen und anderen Sprachen. Berlin; New York: de Gruyter

Weber, Doris (1999): On the Function of Gender. In: Unterbeck, Barbara; Rissanen, Matti (eds): Gender in Grammar and Cognition. Bd. I: Approaches to Gender. Berlin: Mouton de Gruyter. 495-510

Wiese, Bernd (1996): Iconicity and Syncretism. On Pronominal Inllection in Modern German. In: Sackmann, Robin (ed.): Theoretical Linguistics and Grammatical Description. Papers in honour of Hans-Heinrich Lieb. Amsterdam; Philadelphia: Benjamins. 323-344

\author{
Dagmar Bittner \\ University of Potsdam \\ Department of Linguistics \\ Patholinguistics \\ PO Box 601553 \\ D-14415 Potsdam \\ Germany \\ dabitt@zas.gwz-bcrlin.de
}

